\title{
CRISIS Y AUTOGESTIÓN EN EL SIGLO XXI
}

\section{Andrés Ruggeri*}

RESUMENः Cuando en diciembre de 2001 el estallido del modelo neoliberal en Argentina puso los ojos del mundo en los grandes movimientos sociales surgidos o potenciados en el contexto de la enorme crisis que el país atravesaba, activistas y académicos de los países centrales, generalmente vinculados al movimiento antiglobalización, vieron con interés uno de los más llamativos y esperanzadores de esos movimientos, las fábricas ocupadas por sus obreros o, más propiamente, las empresas recuperadas por sus trabajadores. Aunque sabemos que los casos de autogestión obrera en el marco de empresas y fábricas quebradas, vaciadas o abandonadas por sus patrones no carecían de antecedentes históricos, tanto en Argentina como en otras partes del mundo, era la primera vez que se convertían, por lo menos en los últimos decenios, en un movimiento con características e identidad propias.

PALABRAS ClAVE empresas recuperadas por los trabajadores, movimientos sociales, neoliberalismo, autogestión obrera, Argentina.

* Programa Facultad Abierta, Universidad de Buenos Aires.

Este texto es una versión del publicado en Ruggeri, Novaes y Sardá (comps.) (2014). 
ABSTRACT: When in December 2001 the outbreak of the neoliberal model in Argentina put the world's eyes on the great social movements emerged or strengthened in the context of the enormous crisis that the country was facing, activists and academics from the core countries, generally linked to anti-globalization movement, looked forward to one of the most striking and encouraging those movements, factories occupied by their workers or, more properly, companies recovered by their workers. Although we know that cases of worker self-management within companies and factories broken, emptied or abandoned by their employers were not without historical background, both in Argentina and in other parts of the world, it was the first time he became, at least in recent decades, a movement with characteristics and identity.

KEY WORDS: companies recovered by the workers, social movements, neoliberalism, worker self-management, Argentina. 


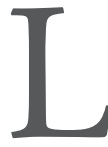

a emergencia de las empresas recuperadas por sus trabajadores (ERT) se daba en un país latinoamericano que, durante los años noventa, había sido el «mejor alumno» del Fondo Monetario Internacional (FMI) y el Banco Mundial, siguiendo al pie de la letra los designios del Consenso de Washington y, de esa manera, ejemplificando con un alto costo social el precio del sometimiento a la absoluta hegemonía del neoliberalismo a nivel mundial. Su existencia también mostraba la esperanza y la posibilidad de generar alternativas a una globalización que parecía ser incontestable después del derrumbe del socialismo soviético y su modelo de economía de planificación centralizada y autoritaria. Intelectuales críticos de los países llamados desarrollados, como los autores del film La Toma, Avi Lewis y Naomi Klein, ${ }^{1}$ pensaban que los casos de autogestión obrera en un país como Argentina podían servir para mostrar la factibilidad de otro camino alternativo a la globalización neoliberal.

¿Cuántos de esos activistas que venían al sur del mundo a ver el raro fenómeno de la autogestión obrera en curso pensaron seriamente que el propio centro capitalista iba a caer en una crisis profunda menos de 10 años después, mientras Argentina y, más ampliamente, Sudamérica, comenzaba a transitar un camino heterodoxo de desarrollo que, sin apuntar a la superación del sistema capitalista, ${ }^{2}$ permitió recuperar los indicadores sociales más importantes y mirar el futuro con esperanza? Los casos de autogestión obrera, que eran vistos casi como un exotismo deseable pero sólo posible en el marco de la crisis de un Estado débil y la economía frágil de un país tercermundista, se convirtieron en una potencial realidad en países de la próspera Unión Europea o en los mismos Estados Unidos, y el ejemplo de las empresas recuperadas comenzó a ser estudiado con otra mirada. La curiosidad inicial pasó a ser reemplazada por la atención sobre algo que puede ocurrir en la propia sociedad.

${ }^{1}$ La Toma, The Take en inglés, es un documental realizado en Argentina durante 2003 mostrando el movimiento de empresas recuperadas, dirigido por Avi Lewis y Naomi Klein. Tuvo un importante papel de difusión del movimiento a escala mundial.

${ }^{2}$ El planteo venezolano por un «socialismo del siglo xxI» es parcialmente acompañado por los gobiernos de Bolivia y Ecuador, no así por Argentina o Brasil. 


\section{CRISIS Y AUTOGESTIÓN EN AMÉRICA LATINA}

En Argentina y en otros países de América Latina la recuperación de empresas por los trabajadores es un proceso que ha ganado notoriedad a pesar de su aún escasa incidencia en términos cuantitativos. Existen empresas recuperadas en casi todos los países sudamericanos y en México, aunque sólo se han constituido como un movimiento de relativa importancia en Uruguay, Brasil y Argentina. En Venezuela, en cambio, los procesos de ocupación y autogestión han ido derivando hacia una suerte de cogestión orientada a partir de políticas del gobierno bolivariano, que en los últimos tiempos han ampliado el horizonte de la participación popular a las colectividades donde se insertan las fábricas, a través de la articulación de consejos de trabajadores y comunas (Azzellini, 2011). En países como México, ${ }^{3}$ el fenómeno está lejos de ser desconocido, pero a pesar de existir grandes cooperativas que derivan de conflictos gremiales, no han adquirido una identidad propia, diferenciada de las cooperativas tradicionales, por lo que el proceso se diluye dentro del cooperativismo o la economía solidaria en general. En Brasil, por su parte, los primeros casos se dan en las décadas de los ochenta y noventa, disminuyendo en años posteriores. Aunque se formaron asociaciones como la Anteag y la Unisol ${ }^{4}$ el panorama muestra dispersión y asimilación al resto de emprendimientos solidarios y cooperativos, como explican Chedid, Novaes y Sardá (2013). ${ }^{5}$ Uruguay y Argentina, por su parte, conforman una realidad más homogénea y es en Argentina donde la crisis de 2001 brindó la amalgama necesaria para que un conjunto de empresas y fábricas ocupadas por cierre y transformadas en empresas autogestionadas conformaran una identidad particular y un movimiento que llamó la atención

${ }^{3}$ México posee una gran tradición de autonomía y autogestión en colectividades rurales, en especial en las áreas indígenas, aunque mucho menos en los movimientos urbanos.

${ }^{4}$ La Asociación Nacional de Trabajadores de Empresas de Autogestión (Anteag) fue la primera asociación de empresas autogestionadas de Brasil, surgida en la primera mitad de los años noventa. La Unisol, filiada a la Central Unitaria de Trabajadores (сUт), se forma ya durante el gobierno de Lula.

${ }^{5}$ Un resumen de este trabajo forma el capítulo 8 en Chedid, Novaes y Sardá (2013). Sardá de Faria (2011) y Novaes (2011) también trabajan el tema y colaboran en esa compilación. 
mundial por su poder simbólico de cuestionamiento a la propiedad privada y a la gestión capitalista de unidades económicas.

Sin explayarnos en el caso argentino, que hemos detallado en varios trabajos (Ruggeri, 2005, 2009, 2011), podemos señalar como característica particular el desarrollo de procesos de autogestión obrera en condiciones muy difíciles y puramente defensivas, como es la resistencia a la pérdida de los puestos de trabajo en condiciones de crisis generalizada, sin apoyo ni contención significativa por parte de partidos políticos, sindicatos o programas estatales. Se trata de procesos autogestionarios que no surgen, en principio, de una voluntad revolucionaria o anticapitalista, sino de la situación de necesidad y abandono en que se encontraron los trabajadores. Esta situación, que llevó a conflictos en torno a la ocupación de los establecimientos, fue derivando - ante la falta de respuestas - en la formación de cooperativas de trabajadores y trabajadoras que, contra toda predicción, tuvieron éxito no sólo en superar el primer y decisivo obstáculo de la reactivación de plantas y establecimientos en condiciones ruinosas y sin capital, sino en llevar adelante procesos de gestión colectiva, sin patrones ni tutela estatal y, lo que es más significativo, sin teoría previa de cómo desarrollar ese camino. Es decir, se trata de una auténtica experiencia obrera llevada a cabo en las difíciles condiciones de una crisis avasalladora, pero al mismo tiempo, sujeta a la creatividad de sus protagonistas para superar una situación estructuralmente sin salida.

También hay que decir que este camino autogestionario fue posible mediante el sostenimiento de poderosas redes de solidaridad social, que aportaron no sólo un apoyo militante clásico, sino innovadoras ideas e iniciativas de apertura de los espacios fabriles a actividades no estrictamente económicas, o por lo menos disímiles y confrontativas con la concepción capitalista de la empresa. Al mismo tiempo, estos casos se enfrentan con la limitante de no conseguir superar algo que está, sin duda, fuera de su alcance, que son las relaciones de intercambio capitalista a las que siguen sometidas como unidades productivas que, nos guste o no, siguen operando como empresas formales en el seno del 
mercado capitalista. Esto, por supuesto, lleva a toda una serie de constricciones y presiones que condicionan la lógica autogestionaria y solidaria interna a los procesos.

Dentro de estas condiciones, las ERT han ido creciendo en cantidad, en número de trabajadores y en actividad económica en los últimos 12 años, como documentan los relevamientos del equipo del programa Facultad Abierta (sumando alrededor de 310 casos y 15,000 trabajadores en la actualidad) (Ruggeri, 2005, 2011, 2013b). Sin embargo, si tenemos en cuenta que en su mayoría las ERT se conforman bajo la figura de cooperativas, ¿por qué nos esforzamos en identificar estos casos en forma diferenciada y qué significación política y económica tiene o puede tener esto?

En nuestra opinión, lo interesante de la empresa recuperada no es solamente el proceso de autogestión que, por diferentes circunstancias, se termina expresando como cooperativa, sino el conjunto del proceso en que una empresa de propiedad privada y gestión capitalista, que explota mano de obra asalariada, pasa a ser de gestión colectiva de los ex asalariados, y los problemas y conclusiones que de ellos se derivan. Problemas, en algunos casos, similares a los del resto de las cooperativas, pero en muchos otros semejantes a aquellos periodos y procesos históricos donde se desarrolló lo que el marxismo del siglo xx llamó «control obrero» y las corrientes anarcosindicalistas prefirieron llamar colectivizaciones o socializaciones. ${ }^{6}$

Insistiremos en la idea de la particularidad del proceso de las ERT frente al movimiento cooperativo en general y a los muy diversos fenómenos de la llamada economía social y solidaria en ciertas cuestiones fundamentales. La primera es, como ya señalamos, el proceso en sí, en el que una unidad económica capitalista, con el funcionamiento jerárquico y vertical que la caracteriza y su finalidad primaria de acumulación de capital a través de la explotación de mano de obra asalariada (sea cual

\footnotetext{
${ }^{6}$ Para un análisis de diferntes procesos históricos de control obrero, véase Mandel (1973) y Ness y Azzellini (2011).
} 
fuere su actividad productiva o de servicios), pasa a ser gestionada en forma colectiva por sus trabajadores. Si bien este proceso puede ser identificado en el origen de muchas cooperativas históricas, incluso en los mismos inicios del cooperativismo, se diferencia de la formación de empresas cooperativas sin un origen previo de gestión privada no sólo en el traspaso de la gestión vertical a la colectiva sino en el propio hecho de la socialización de la propiedad de los medios de producción. Aun sin que ello signifique una generalización de este proceso de socialización o colectivización, lleva en sí mismo un cuestionamiento profundo a los fundamentos de la gestión capitalista de la economía y la sociedad.

La segunda razón importante para diferenciar las ERT de otros casos cooperativos es la clara pertenencia de la experiencia a la lucha de la clase trabajadora. Podemos argumentar que en todas o la mayoría de las cooperativas y emprendimientos de economía solidaria hay protagonismo de trabajadores, pero encontramos una sustancial diferencia entre la mayoría de éstas y un proceso conflictivo originado en el corazón de las contradicciones entre el capital y el trabajo (el abandono patronal y la ocupación o el conflicto desatado a partir de ese abandono o cierre compulsivo). Por otro lado, esa diferencia forma parte de la propia autopercepción de los protagonistas de la recuperación como trabajadores antes que como cooperativistas o como «excluidos», identidad difusa donde se pierde la noción de pertenencia a la clase que vive del trabajo. ${ }^{7}$ La recuperación de empresas y fábricas por los trabajadores como método extremo de defensa del empleo, y luego como autoafirmación de la propia identidad como trabajadores sin patrón, pone también en cuestión las formas de organización y las herramientas tradicionales de lucha del movimiento obrero, vale decir, el papel de los sindicatos, partidos políticos clasistas y, también, de las propias cooperativas, mayoritariamente alejadas en el presente del viejo origen común con los movimientos proletarios.

\footnotetext{
${ }^{7}$ Una crítica desde esta óptica al concepto de exclusión se puede ver en Trinchero (2009).
} 
Esto es, en otras palabras, retomar el camino originario del movimiento cooperativo como un movimiento de acción de los trabajadores en la formación de una lógica económica no capitalista, en la que no sólo deben respetarse los principios solidarios y autogestionarios, sino que debe quedar bien en claro la negativa a la explotación capitalista de asalariados.

\section{DE CRISIS EN CRISIS}

Para los argentinos o sudamericanos que vivimos la crisis como algo repetido en la vida de las últimas generaciones, la situación de países como España o Grecia parece una película ya vista. Una crisis provocada por el capital financiero que es empeorada - con el argumento de ser la única manera de enfrentarla - siguiendo las viejas y conocidas recetas de «austeridad» que dejan en el desempleo a millones de trabajadores, recortan el presupuesto destinado a la protección social, obligan a los Estados a desprenderse de las empresas públicas y recortan gastos en el armazón del «Estado de bienestar» trabajosamente creado décadas atrás. Y, como reverso de la trama, se destinan enormes presupuestos a fortalecer los aparatos de represión de la inevitable protesta social, se facilita la movilidad extrema del capital en búsqueda de mejores condiciones de explotación y precarización del trabajo y las máximas facilidades para la acumulación del capital, se destinan cifras nunca antes vistas de fondos públicos para el «rescate» de las entidades bancarias responsables de la crisis y se castiga a quienes intenten una respuesta diferente.

La diferencia entre una crisis con estas características en la periferia del sistema capitalista y una en el centro del capitalismo global es, justamente, la centralidad de las economías que se ven afectadas, la fortaleza de las instituciones y herramientas financieras en juego, la fortaleza de la hegemonía ideológica y cultural capitalista, la fuerza represiva e institucional de los Estados y su capacidad de respuesta como custodios de los intereses más profundos del capital. Los movimientos obreros y 
populares de estos países, a su vez, parecen estar en peores condiciones para la resistencia, paradójicamente a causa de los múltiples mecanismos de protección social que habían logrado conquistar en los decenios anteriores, debilitando a la vez los anticuerpos de lucha y organización obrera que permitirían una reacción rápida y costosa para la gobernabilidad capitalista. El conformismo y resignación sobre qué cambios eran posibles, inclusive entre la versión más reformista de los partidos socialdemócratas mayoritarios de la izquierda europea, disminuidos a adaptarse y convertirse en la versión «humana» del capitalismo neoliberal global, contribuyó no poco a esa indefensión y decepción en sus bases.

En los últimos dos o tres años han proliferado los conflictos laborales y el cierre de empresas y establecimientos productivos en la mayoría de los países de Europa y también en Estados Unidos. El caso de la fábrica de puertas y ventanas Republic, en Chicago, fue uno de los más conocidos en América del Norte. Sin embargo, es mucho más importante el proceso de recuperación de empresas en los países del sur de Europa, los más afectados por la crisis. Casos como el de Vio.Me, en Tesalónica, Grecia, están ocupando el papel que supieron tener en el activismo Zanón o IMPA, por citar las empresas recuperadas argentinas más famosas del periodo inmediatamente posterior a diciembre de 2001. Otros ejemplos son menos conocidos, incluso entre la militancia de la izquierda radical de sus propios países, como varias cooperativas que bien podemos catalogar como empresas recuperadas en el Estado español, especialmente en Cataluña, u otras en distintos lugares de Francia, algunas de ellas con varios años de funcionamiento. En Italia, por su parte, la ley habilita la formación de cooperativas a partir de procesos de quiebra. Pero también allí, como es la norma, cuando estas quiebras son repentinas y en muchos casos fraudulentas, las dificultades efectivas para que los trabajadores puedan hacerse cargo de las empresas son grandes y tortuosas, y hay distintas apreciaciones sobre las dimensiones e incluso la identidad del proceso.

Algunos investigadores mencionan la existencia de varias decenas de ERT, pero los casos que se autoadscriben en esa categoría son hasta 
el momento más bien escasos, como la romana Officine Zero y la milanesa Rimaflow. ${ }^{8}$ Se trata, en estos dos casos, de fábricas cerradas con muy escasas posibilidades de reactivación productiva en su actividad anterior, y que al ser ocupadas por parte de sus antiguos trabajadores $y$ un conjunto heterogéneo de activistas sociales y políticos tienden más a recrear el ejemplo argentino de la «fábrica abierta», sede de múltiples actividades y emprendimientos solidarios, culturales y laborales, sin muchas posibilidades de lograr una recuperación como unidad industrial. Afinando un poco más la mirada, podemos ver algunas particularidades de estos aún poco conocidos casos europeos de empresas recuperadas por los trabajadores. La primera es que, a diferencia de los latinoamericanos, los europeos parecen tener mayores dificultades en la construcción de redes solidarias de sostenimiento y apoyo a las experiencias (aunque casos como los anteriormente nombrados deben gran parte de sus posibilidades de sobrevivencia a la existencia misma de estas redes). Las instituciones de seguridad social todavía existentes provocan que la situación de los trabajadores al cierre de las plantas no sea terminal, como ocurre mayoritariamente en Argentina y en América Latina en general. Los seguros de desempleo (el «paro», en España) permiten que los trabajadores tengan un ingreso regular durante un tiempo determinado, que demora el momento en que los ex asalariados se enfrenten a la circunstancia de estar sin ingreso alguno y, por lo tanto, desalienta el tener que recurrir a métodos de lucha radicales para conservar el trabajo.

La percepción de la profundidad de la crisis y la gravedad de la situación del desempleo crónico estructural también es diferente, especialmente en los primeros tiempos de la crisis, y la idea de que el deterioro económico será un fenómeno pasajero está, consciente o inconscientemente, detrás de la falta de reacción de los trabajadores al

\footnotetext{
${ }^{8}$ Aunque no hay todavía investigaciones publicadas sobre la cuestión, podemos citar comunicaciones con investigadores como Vieta, que habla de cerca de 100 ERT en el territorio italiano, y Carrano, que menciona a unas 30. Sobre el caso Rimaflow, véase el artículo de Semanzin y Magnani (2013).
} 
momento de la pérdida del empleo. La complicidad o la falta de visión de los sindicatos mayoritarios también aportó (en este caso de forma parecida a América Latina) a la pasividad del movimiento obrero, en baja desde décadas anteriores y sin capacidad de reacción, en parte debida a lealtades políticas con los partidos socialdemócratas en el gobierno en algunos países. La falta de reacción en el momento de cierre empresarial y la prolongada supervivencia ${ }^{9}$ con base en los seguros de desempleo, junto con la amplia hegemonía ideológica del capital, estructurada especialmente en torno al proyecto económico de la Unión Europea, parece demorar notablemente los procesos de toma de conciencia de la situación real. Para el tiempo en que el pago de las prestaciones llega a su fin, no sólo los establecimientos están ya definitivamente cerrados, sino que también los colectivos de trabajo se encuentran absolutamente disueltos, librando a cada trabajador a la salida individual.

Sin embargo, hay otra característica que diferencia los casos europeos de los sudamericanos, relacionada con la naturaleza de la crisis. En varios de los ejemplos más conocidos, como la francesa Fralib, fábrica de té en las inmediaciones de Marsella, o en la italiana Rimaflow, el cierre empresarial se debió no a una quiebra genuina sino a una decisión empresarial de aprovechar las ventajas del menor costo de la fuerza de trabajo en otras regiones del mundo, en particular en países del Este europeo. El cierre de ambas plantas no se debió, como en la mayoría de los casos argentinos o brasileños, a una decisión de acabar con la actividad productiva de la empresa por incapacidad de llevar adelante el negocio o por la conveniencia de pasar los activos al mercado financiero, sino a un traslado a (en este caso) Polonia, donde el mismo tipo de producción implica un menor costo impositivo y, especialmente, laboral, que compensa con creces el aumento del costo de distribución de la misma producción en los mismos mercados. El caso de Fralib, planta perteneciente a la multinacional Unilever, es especialmente revelador de esta dinámica, en la cual podemos ver que «la crisis» no es necesariamente el

\footnotetext{
${ }^{9}$ Según los países, varía entre seis meses y dos años.
} 
problema, sino un buen pretexto para acrecentar la movilidad extrema del capital en búsqueda de las condiciones de mayor rentabilidad y acumulación a expensas de los derechos de los trabajadores y de pasar por alto las viejas regulaciones nacionales. La transnacionalización del marco regulatorio deja atrás y sin sentido una importante cantidad de legislaciones conquistadas por el movimiento obrero en décadas pasadas, reemplazadas por normativas europeas o internacionales que las dejan obsoletas y sin marco posible de reacción para una clase trabajadora que perdió toda capacidad de acción en el plano internacional, mientras que el capital, a la inversa, ganó movilidad y capacidad de legislar a su favor a nivel mundial, un signo de los tiempos de la globalización.

Este tipo de problemáticas, que en países como Argentina todavía se suelen expresar en términos de la contradicción centro-periferia (por la cual la producción nacional es reemplazada por productos importados, provenientes de transnacionales que operan en países con costos bajísimos de la fuerza de trabajo, e imposición de políticas por los organismos financieros globales a través de mecanismos de desregulación/regulación internacionales y el manejo de los créditos y deuda externas), en el plano europeo muestran cómo la formación de un supragobierno del capital financiero, expresado por la Troika (Banco Central Europeo, Fmi y Comisión Europea) que impone planes de ajuste, recortes impensables poco tiempo antes a salarios, inversión pública, desmantelamiento de la seguridad social y empeoramiento de las condiciones de trabajo, es la causa de un cierre programado de la producción basado en razones de búsqueda de una mayor rentabilidad y no de imposibilidad de competir o producir por culpa de la crisis. Al contrario, la crisis es el contexto ideal para que empresas transnacionales que no tienen problemas productivos ni financieros, ni dificultades para colocar su producción en el mercado local (el caso de Fralib es ejemplar, el mismo producto se sigue vendiendo en Francia, pero traído ahora desde Polonia), cierren plantas en países donde no sólo tenían mayores regulaciones laborales, sino mejores salarios y sindicatos con cierto poder, y las relocalicen en regiones que ofrezcan mayores 
condiciones para la acumulación capitalista a costa de sus trabajadores. La crisis es, entonces, una oportunidad para que la gran burguesía europea, bajo el comando de los intereses financieros encarnados por los bancos centrales de las mayores potencias económicas, empiece a desarmar con celeridad los mecanismos, regulaciones y el mismo armazón del Estado de bienestar que debieron en su momento conceder a los poderosos sindicatos y partidos obreros que emergieron con fuerza después de la segunda guerra mundial, temerosos ante el peligro de la expansión del comunismo de tipo soviético.

La recuperación de empresas por parte de los trabajadores se convierte así, en estos países, en un proceso quizá más arduo que en las economías sudamericanas o de países del tercer mundo en general, pues los gobiernos no tienen por el momento mayor interés - ni tampoco se ven en la necesidad- de ceder ante los trabajadores en circunstancias donde vienen demostrando que aún no llegaron al punto en que les importe demasiado el costo social de sus políticas. También la escasa articulación de redes de apoyo, la incomprensión mayoritaria de sus sindicatos y la aún inexistente conformación de un movimiento por la autogestión (a lo que contribuye la fuerza y la indiferencia simultáneas de las organizaciones cooperativistas tradicionales), esboza un marco de dificultad importante. Pero, por otra parte, la ventaja de contar con el ejemplo vivo de otros lugares del mundo y de la existencia de amplios sectores sociales que no sólo no están acostumbrados a la crisis (a vivir miserablemente), sino que pueden potencialmente ver muy claro cuáles son los riesgos a los que se exponen de seguir avanzando la situación de desmantelamiento de los logros de las generaciones anteriores, puede ser un factor importante de movilización y organización. Junto con eso, una mayor posibilidad de respuesta económica y de reorientar redes de apoyo mayoritariamente construidas para la solidaridad externa a la propia sociedad (siempre y cuando se logre transformar el voluntariado en militancia) pueden dar un marco de organización que fortalezca las experiencias y permita un avance en la interacción entre teoría y práctica de la autogestión. 
Quizá esta descripción de la dinámica de la explotación capitalista internacional del trabajo y la movilidad cada vez mayor de la transnacionalización del capital parezca poco relacionado con el tema de este artículo alrededor de las luchas por la autogestión. Sin embargo, es fundamental para entender la lógica por la cual los capitalistas deciden abandonar establecimientos productivos eficientes e, incluso, de última tecnología, en lugares donde el mercado para esos productos no sólo no ha desaparecido, sino que en algunos casos hasta se expande. Esa lógica funciona mientras los trabajadores de los países de origen de las transnacionales no resistan el cierre, por un lado (y la autogestión obrera es no sólo una forma de mantener la fuente laboral y de avanzar hacia otra lógica de producción, sino también una forma de resistencia frente a los abusos del capital), y mientras los trabajadores de los países del tercer mundo no logren mejorar sus condiciones de trabajo y elevar sus salarios. O, en el mejor de los casos, luchar también por la autogestión de sus fábricas, restringiendo de este modo la movilidad impune del capital.

El caso argentino, a pesar de su aún escaso número, muestra que cuando los trabajadores asumen la autogestión de las empresas abandonadas como una herramienta de lucha, defensiva y ofensiva a la vez, mejoran las condiciones laborales de todos, los autogestionados y los asalariados, $y$ los patrones empiezan a sentir que alguien, por una vez en los últimos decenios, les pone límites. Y si ya no es el Estado de compromiso interclasista, también llamado Estado de bienestar, el que pone esos límites, van a tener que ser los trabajadores.

Estas cuestiones colocan a las experiencias sudamericanas - especialmente las argentinas, por su número y por el hecho de haberse constituido como un movimiento específico de autogestión (más allá de la fragmentación organizativa y política que les impide mostrarse como un todo orgánico) - en un eje articulador que permite reinstalar en el debate de la izquierda mundial el problema de la autogestión. La hegemonía del pensamiento económico y la práctica política de las corrientes comunistas y socialdemócratas en el siglo $\mathrm{xx}$, especialmente después de 
la revolución rusa y la derrota de los anarquistas españoles en la década de los treinta dejaron prácticamente fuera del debate político y económico mundial la opción autogestionaria, con algunas excepciones notorias. ${ }^{10} \mathrm{El}$ fracaso contundente del socialismo de tipo soviético (incluso en aquellos países donde los partidos comunistas retuvieron el poder después de la caída de la Unión de Repúblicas Soviéticas Socialistas, URSS), ${ }^{11}$ en especial en el aspecto económico donde deberían haber probado (de acuerdo con la teoría y la creencia del siglo xix y principios del $\mathrm{xx}$ ) su superioridad frente al capitalismo, es decir, en la capacidad de proveer más o menos satisfactoria e igualitariamente a las necesidades del conjunto de la sociedad, abrió paso a una etapa donde esas teorías hegemónicas de la izquierda se enfrentaron a sus propios límites.

Esta nueva etapa llevó a que los movimientos sociales de clase trabajadora de las últimas dos décadas se empezaran a plantear, generalmente por la vía de los hechos, la alternativa autogestionaria. Y así como esto se fue dando primero en la práctica y luego en la teoría, las experiencias de empresas recuperadas, comunas autónomas, redes de intercambio no monetarias o alternativas, comienzan a tener peso en la reconstitución del nuevo pensamiento anticapitalista. Es en el área económica donde el capitalismo muestra su fortaleza y a la vez su debilidad, y es en esta área donde estamos todavía bastante huérfanos de teoría, lo cual es imperdonable existiendo ya experiencias concretas con cierto desarrollo para estudiar, analizar y extraer conclusiones.

De esta manera, las empresas recuperadas y autogestionadas cumplen un papel que va mucho más allá de la ya de por sí valiosa defensa

\footnotetext{
${ }^{10}$ En la segunda posguerra mundial, las huelgas del Mayo francés, el movimiento de autonomía obrera italiano, la experiencia yugoslava y los cordones industriales del Chile de Allende son los momentos más importantes en los que el debate sobre la autogestión y el control obrero retomaron protagonismo.

${ }^{11}$ Con la relativa excepción de Cuba, en donde el modelo económico heredado de la alianza con la urss ha sido puesto en debate, con mayor o menor profundidad, en numerosas ocasiones (durante los años sesenta, a mediados de los ochenta y en los noventa) y, en la actualidad, en que se vive un proceso de cambio en el que las cooperativas aparecen como una opción de renovación de la organización productiva. Véase Piñeiro Harnecker (2011).
} 
y recuperación de puestos de trabajo: dan el puntapié inicial para la reformulación, reconstrucción o directamente nueva constitución de una teoría y una praxis económica de los trabajadores que logre superar los esquemas tradicionales (o impuestos como camino más o menos único a lo largo del siglo $\mathrm{xx}$ ) y problematizar la cuestión de la autogestión en una dimensión que hasta el momento no se había llevado a cabo o lo fue en forma marginal. En ese sentido, en la medida en que la recuperación de empresas y la autogestión dejen de ser fenómenos particulares de determinados países y coyunturas históricas, y pasen a ser una estrategia de lucha internacional de la clase trabajadora (tan internacional como el movimiento predatorio del capital), la posibilidad de reconstruir el proyecto de una sociedad y economía poscapitalista, que reinvente el viejo proyecto socialista, que reconozca que las diversas tradiciones de la izquierda y los movimientos populares de los siglos xix y $\mathrm{xx}$ en distintas partes del mundo tienen algo que aportar, va a ser posible.

\section{BIBLIOGRAFÍA}

AA.vv. (1969), Congresos de las internacionales socialistas, selección de documentos, Buenos Aires, Siglomundo, CEAL.

Azzellini, Dario (2011), «De las cooperativas a las empresas de propiedad social directa en el caso venezolano», en Camila Piñeiro Harnecker (comp.), Cooperativas y socialismo: una mirada desde Cuba, La Habana, Editorial Caminos.

Bowman y Stone (2009), «La cooperativización como alternativa al capitalismo globalizador», en A. Ruggeri (coord.), La economía de los trabajadores: autogestión y distribución de la riqueza, Buenos Aires, Ediciones de la Cooperativa Chilavert.

Chedid Henriques et al. (2013), Empresas Recuperadas por Trabalhadores no Brasil, Río de Janeiro, Editora Multifoco.

Cole, GdH (1957), Historia del pensamiento socialista, tomo I, Los precursores, 1789-1856, México, Fondo de Cultura Económica.

(1959), Historia del pensamiento socialista, tomo III, La Segunda Internacional 1889-1914, México, Fondo de Cultura Económica. 
Gómez Solórzano, Marco A. (2014), «Trabajo Precario Global», ponencia presentada al IV Encuentro Internacional «La Economía de los Trabajadores», Joao Pessoa, Paraiba. Brasil.

ICA Explorative World Report (2012), Exploring the Co-operative Economy, en http://ica.coop/sites/default/files/attachments/Explorative_Report_2012. pdf

Mandel, Ernest (1973), Control obrero, consejos obreros, autogestión, Buenos Aires, Ediciones La Ciudad Futura.

Ness y Azzellini (2011), Ours to Master and to Own, Workers' Control form the Commune to the Present, Chicago, Haymarket Books.

Ngai Pun, Jenny Cham y Mark Selden (2013), «The Politics of Global Production: Apple, Foxconn and China's New Working Class», New Technology, Work and Employment, Rev. 2, julio de 2013.

Novaes, Henrique T. (2011), O retorno do caracol a sua concha: alienaçao e desalienaçao em associacioes de trabalhadores, Sao Paulo, Editora Expressao Popular.

Organizaciones Piqueteras, Buenos Aires, Biblos.

Piñeiro Harnecker, Camila (comp.) (2011), Cooperativas y socialismo: una mirada desde Cuba, La Habana, Editorial Caminos.

Ruggeri, Andrés (2009a), Las empresas recuperadas: autogestión obrera en Argentina y América Latina, Buenos Aires, Editorial de la Facultad de Filosofía y Letras.

(2009b), La economía de los trabajadores: autogestión y distribución de la riqueza, Buenos Aires, Ediciones de la Cooperativa Chilavert.

Ruggeri et al. (2011), «Las empresas recuperadas en la Argentina. 2010», Informe del tercer relevamiento de empresas recuperadas, Buenos Aires, Ediciones de la Cooperativa Chilavert.

Ruggeri, Andrés (2012), «Historia de la autogestión. Los comienzos», Revista Autogestión, vol. XXI, núm. 1.

Ruggeri, Andrés (2013a), «Historia de la autogestión. Los pioneros de Rochdale», Revista Autogestión, vol. XXI, núm. 2.

Ruggeri et al. (2013b), «Las nuevas empresas recuperadas en la Argentina (20102013)», Informe del cuarto relevamiento de empresas recuperadas, Buenos Aires, Ediciones de la Cooperativa Chilavert.

Ruggeri, A., C. Martínez y H. Trinchero (2005), «Las empresas recuperadas en la Argentina», Informe del segundo relevamiento de empresas recuperadas por los trabajadores, Buenos Aires, Facultad de Filosofía y Letras-ubA. 
Ruggeri, Novaes y Sardá (comp.) (2014), Crisis y autogestión en el siglo XXI. Cooperativas y empresas recuperadas en tiempos de neoliberalismo, Buenos Aires, Ediciones Peña Lillo/Continente.

Sacchetto, Devi y Marco Semenzin (2013), «Workers' Cooperatives in Italy Between Solidarity and Autocratic Centralism», International Forum Rethinking Economy: Social/Solidarity Economy in China and the World, Peking U. PolyU China Social Work Research Centre.

Sardá de Faria, Mauricio (2011), Autogestão, Cooperativa, Economia Solidária: avatares do trabalho e do capital, Florianópolis, Editora Em Debate, Universidade Federal de Santa Catarina.

Semenzin, Marco y Esteban Magnani (2013), «El movimiento de autogestión liderado por trabajadores llegó a Italia», en http://www.pagina12.com.ar/diario/suplementos/cash/17-6846-2013-05-26.html.

Trinchero, Héctor Hugo (2009), «De la exclusión a la autogestión: innovación social desde las empresas recuperadas por sus trabajadores (ERT)», en A. Ruggeri, La economía de los trabajadores: autogestión y distribución de la rique$z a$, Buenos Aires, Ediciones de la Cooperativa Chilavert. 